\title{
DECENTRALISED SLIDING MODE CONTROL FOR NONMINIMUM PHASE NONLINEAR INTERCONNECTED SYSTEMS
}

\author{
Xing-Gang Yan*, Sarah K. Spurgeon*, Christopher Edwards* \\ ${ }^{*}$ Control $\&$ Instrumentation Research Group \\ Department of Engineering \\ University of Leicester, LE1 7RH, U.K. \\ e-mail:xy3@le.ac.uk, eon@le.ac.uk,ce14@le.ac.uk
}

\begin{abstract}
A class of interconnected systems with nonlinear interconnections and nonlinear disturbances is considered. A continuous nonlinear reduced-order compensator is established by exploiting the structure of the uncertainties. A sliding surface is proposed in an augmented space formed by the system output and the compensator variables, and the stability of the corresponding sliding mode is analysed. Then, a robust decentralised dynamical output feedback sliding mode controller is designed to drive the system to the composite sliding surface and maintain a sliding motion on it thereafter. Copyright ${ }^{(} 2005$ IFAC.
\end{abstract}

Keywords: nonlinear interconnected system, sliding modes, nonminimum phase

\section{INTRODUCTION}

Sliding mode techniques are employed to study the stabilization of a class of nonlinear interconnected systems. Mismatched uncertainties and nonlinear interconnections are considered, and the bounds on the uncertainties take more general forms as in (Yan et al. 2004), (Yan and Xie 2003). By using the structure of the uncertainties, a continuous reduced-order compensator is proposed based on constrained Lyapunov equations. Then, a sliding surface is proposed in the augmented space formed by the compensator and system output. Using an equivalent control approach and a local coordinate transformation, the sliding mode dynamics are established and the stability is analysed. A robust decentralised output feedback sliding mode control scheme is synthesized such that the interconnected system can be driven to the pre-designed sliding surface. This approach al-

\footnotetext{
1 The authors acknowledge the support of the EPSRC
} (Grant reference GR/R32901/01). lows both the nominal isolated subsystem and the whole nominal system to be nonminimum phase. It should be emphasised that methods to deal with nonlinear interconnections are a key issue in the control of interconnected systems. So far nearly all associated work treats such interconnections as disturbances and then uses an extra stability margin to reject the effect of the interconnections. By dealing with uncertain interconnections and known interconnections separately, the conservatism is reduced to some extent as claimed in (Yan and Xie 2003). However, the interconnections are still treated as a disturbance in the sense that the interconnections are not used explicitly in the control design. In this paper, it is shown that by employing sliding mode techniques, the interconnections are directly used in the control design, which together with the fact that the sliding mode dynamics are reduced-order systems, reduces conservatism and enhances robustness.

Notation: For a square matrix $A, \underline{\lambda}(A)$ and $\bar{\lambda}(A)$ denote the minimum and maximum eigenvalues 
respectively. $A>0$ means that $A$ is positive definite. $I_{n}$ denotes the unit matrix with dimension $n$. The set of $n \times m$ matrices with elements defined in $\mathbb{R}$ will be denoted by $\mathbb{R}^{n \times m}$. For a function/vector $f(x), L_{f}$ denote its Lipschitz constant in an associated domain. $\|\cdot\|$ denotes the Euclidean norm or its induced norm.

\section{SYSTEM DESCRIPTION}

Consider a nonlinear interconnected system composed of $N$ subsystems as follows

$$
\begin{aligned}
\dot{x}_{i}= & A_{i} x_{i}+B_{i} u_{i}+\Delta f_{i}\left(x_{i}\right)+ \\
& \sum_{\substack{j=1 \\
j \neq i}}^{N}\left(H_{i j}\left(x_{j}\right)+\Delta H_{i j}\left(x_{j}, t\right)\right), \\
y_{i}= & C_{i} x_{i}, \quad i=1,2, \ldots, N,
\end{aligned}
$$

where $x_{i} \in \Omega_{i} \subset \mathbb{R}^{n_{i}}\left(0 \in \Omega_{i}\right), u_{i} \in \mathbb{R}^{m_{i}}$ and $y_{i} \in \mathbb{R}^{p_{i}}$ are the states, inputs and outputs of the $i$-th subsystem respectively with $m_{i}<n_{i}$; $\left(A_{i}, B_{i}, C_{i}\right)$ are constant matrices of appropriate dimensions with $B_{i}$ and $C_{i}$ of full rank; $\Delta f_{i}$ is the mismatched uncertainty of the $i$-th isolated subsystem, $\sum_{\substack{j=1 \\ j \neq i}}^{N} H_{i j}$ and $\sum_{\substack{j=1 \\ j \neq i}}^{N} \Delta H_{i j}$ are respectively the known and the uncertain interconnections of the $i$-th subsystem with $H_{i j}(0)=0$. The functions are all assumed to be continuous in their arguments.

Without loss of generality, suppose that the nonlinear functions $H_{i j}(\cdot)$ have decompositions

$$
\begin{aligned}
H_{i j}\left(x_{j}\right)= & \Phi_{i j}\left(x_{j}\right) x_{j}, \\
& i \neq j, \quad i, j=1,2 \ldots, N
\end{aligned}
$$

where $\Phi_{i j}(\cdot)$ are continuous. The decomposition (3) is always true for $H_{i j}(\cdot)$ smooth enough in their domain of definition satisfying $H_{i j}(0)=0$.

In order to facilitate the analysis,

- All equations and inequalities involving the indexes $i$ and/or $j$ are satisfied for all $i, j=$ $1,2, \ldots, N(i \neq j)$;

- The considered domain is

$$
\begin{aligned}
x & =\operatorname{col}\left(x_{1}, x_{2}, \ldots, x_{N}\right) \in \Omega \\
& \equiv: \Omega_{1} \times \Omega_{2} \times \cdots \times \Omega_{N}
\end{aligned}
$$

with $x_{i} \in \Omega_{i} \subset \mathbb{R}^{n_{i}}$;

- Output matrices

$$
C_{i}=\left[\begin{array}{ll}
I_{p_{i}} & 0
\end{array}\right]
$$

Assumption 1. The matrix pairs $\left(A_{i}, B_{i}\right)$ and $\left(A_{i}, C_{i}\right)$ are controllable and detectable respectively, and the function $H_{i j}\left(x_{j}\right)(i \neq j)$ satisfies Lipschitz conditions in the considered domain.

In view of the detectability of $\left(A_{i}, C_{i}\right)$, there exists a matrix $L_{i}$ such that $\left(A_{i}-L_{i} C_{i}\right)$ is stable and thus for any $Q_{i}>0$ the following Lyapunov equation has a unique solution $P_{i}>0$

$$
\left(A_{i}-L_{i} C_{i}\right)^{\tau} P_{i}+P_{i}\left(A_{i}-L_{i} C_{i}\right)=-Q_{i}
$$

Assumption 2. The uncertainties have structural decompositions of the following form

$$
\begin{gathered}
\Delta f_{i}\left(x_{i}, t\right)=D_{i} \Delta \widetilde{f}_{i}\left(x_{i}, t\right), \\
\Delta H_{i j}\left(x_{j}, t\right)=E_{i j} \Delta \widetilde{H}_{i j}\left(x_{j}, t\right)
\end{gathered}
$$

where $D_{i}, E_{i j}(i \neq j)$ are constant matrices, and

$$
\begin{aligned}
\left\|\Delta \widetilde{f}_{i}\left(x_{i}, t\right)\right\| & \leq \rho_{i}\left(y_{i}, t\right) \gamma_{i}\left(x_{i}, t\right), \\
\left\|\Delta \widetilde{H}_{i j}\left(x_{j}, t\right)\right\| & \leq \vartheta_{i j}\left(y_{j}, t\right) \zeta_{i j}\left(x_{j}, t\right)
\end{aligned}
$$

where $\gamma_{i} \leq \widetilde{\gamma}_{i}\left(x_{i}, t\right)\left\|x_{i}\right\|$ and $\zeta_{i j} \leq \widetilde{\zeta}_{i j}\left(x_{j}, t\right)\left\|x_{j}\right\|$ $(i \neq j)$ are Lipschitz with $\widetilde{\gamma}_{i}$ and $\widetilde{\zeta}_{i j}$ continuous.

Assumption 3. There exist matrices $G_{i}$ and $F_{i j}$ $(i \neq j)$ such that

$$
D_{i}^{\tau} P_{i}=G_{i} C_{i}, \quad E_{i j}^{\tau} P_{i}=F_{i j} C_{i}
$$

where $P_{i}$ satisfies (4) and the matrices $D_{i}, E_{i j}$ $(i \neq j)$ satisfy $(5)$.

It should be noted that Assumption 3 implies that

$$
\begin{aligned}
& \operatorname{rank}\left(D_{i}^{\tau} P_{i}\right)=\operatorname{rank}\left(\left[\begin{array}{ll}
D_{i}^{\tau} P_{i} & C_{i}
\end{array}\right]\right) \\
& \operatorname{rank}\left(E_{i j}^{\tau} P_{i}\right)=\operatorname{rank}\left(\left[\begin{array}{ll}
E_{i j}^{\tau} P_{i} & C_{i}
\end{array}\right]\right)
\end{aligned}
$$

with $i, j=1,2, \ldots, N \quad(i \neq j)$.

The objective of this paper is to use sliding mode techniques to develop an output feedback control scheme based on a continuous reducedorder compensator such that the corresponding closed-loop system is asymptotically stable.

\section{COMPENSATOR DESIGN}

Consider system (1)-(2). Following the partition of $C_{i}=\left[\begin{array}{ll}I_{p_{i}} & 0\end{array}\right]$, the system can be rewritten

$$
\begin{gathered}
{\left[\begin{array}{c}
\dot{x}_{i 1} \\
\dot{x}_{i 2}
\end{array}\right]=\left[\begin{array}{ll}
A_{i 1} & A_{i 2} \\
A_{i 3} & A_{i 4}
\end{array}\right]\left[\begin{array}{l}
x_{i 1} \\
x_{i 2}
\end{array}\right]+\left[\begin{array}{l}
B_{i 1} \\
B_{i 2}
\end{array}\right] u_{i}+} \\
{\left[\begin{array}{c}
D_{i 1} \\
D_{i 2}
\end{array}\right] \Delta \widetilde{f}_{i}+\left[\begin{array}{l}
\sum_{\substack{j=1 \\
j \neq i}}^{N}\left(H_{i j 1}+E_{i j 1} \Delta \widetilde{H}_{i j}\right) \\
\sum_{\substack{j=1 \\
j \neq i}}^{N}\left(H_{i j 2}+E_{i j 2} \Delta \widetilde{H}_{i j}\right) \\
y_{i}=x_{i 1}
\end{array}\right]}
\end{gathered}
$$

where $x_{i}=\operatorname{col}\left(x_{i 1}, x_{i 2}\right)$ with $x_{i 1} \in \mathbb{R}^{p_{i}}, A_{i 1} \in$ $\mathbb{R}^{p_{i} \times p_{i}}, B_{i 1} \in \mathbb{R}^{p_{i} \times m_{i}} ; D_{i 1}, E_{i j 1}$ and $H_{i j 1}$ are the first $p_{i}$ rows of $D_{i}, E_{i j}$ and $H_{i j}\left(x_{j}\right)$ respectively. Partition $P_{i}, Q_{i}$ and $L_{i}$ conformably with the decomposition (8)-(9) as

$$
\begin{aligned}
P_{i} & =\left[\begin{array}{ll}
P_{i 1} & P_{i 2} \\
P_{i 2}^{\tau} & P_{i 3}
\end{array}\right], Q_{i}=\left[\begin{array}{ll}
Q_{i 1} & Q_{i 2} \\
Q_{i 2}^{\tau} & Q_{i 3}
\end{array}\right] \\
L_{i} & =\left[\begin{array}{l}
L_{i 1} \\
L_{i 2}
\end{array}\right]
\end{aligned}
$$

Then, construct a dynamical system 


$$
\begin{aligned}
& \dot{\hat{z}}_{i 2}=\left(A_{i 4}+P_{i 3}^{-1} P_{i 2}^{\tau} A_{i 2}\right) \hat{z}_{i 2}+\left(P_{i 3}^{-1} P_{i 2}^{\tau}\left(A_{i 1}-A_{i 2} P_{i 3}^{-1} P_{i 2}^{\tau}\right)\right. \\
& \left.+A_{i 3}-A_{i 4} P_{i 3}^{-1} P_{i 2}^{\tau}\right) y_{i}+\left(P_{i 3}^{-1} P_{i 2}^{\tau} B_{i 1}+B_{i 2}\right) u_{i}+ \\
& \sum_{\substack{j=1 \\
j \neq i}}^{N}\left[P_{i 3}^{-1} P_{i 2}^{\tau} H_{i j 1}\left(y_{j}, \hat{\nu}_{j}\right)+H_{i j 2}\left(y_{j}, \hat{\nu}_{j}\right)\right]
\end{aligned}
$$

where $\hat{\nu}_{j}=\hat{z}_{j 2}-P_{j 3}^{-1} P_{j 2} y_{j}$ and $\hat{z}_{i 2} \in \mathbb{R}^{n_{i}-p_{i}}$. The following conclusion can be drawn:

Theorem 1. Let $\hat{x}_{i 2}=-P_{i 3}^{-1} P_{i 2}^{\tau} y_{i}+\hat{z}_{i 2}$ with $\hat{z}_{i 2}$ as given above. Then, under Assumptions 1-3 there exist positive constants $\alpha_{1}$ and $\alpha_{2}$ such that

$$
\left\|x_{i 2}(t)-\hat{x}_{i 2}(t)\right\| \leq \alpha_{1} \exp \left\{-\alpha_{2} t\right\}
$$

if $W^{T}+W$ is positive definite with $W=\left(w_{i j}\right)_{N \times N}$ defined by

$$
w_{i j}=\left\{\begin{array}{cc}
\underline{\lambda}\left(Q_{i 3}\right), & i=j \\
-2\left(\left\|P_{i 2}\right\| L_{H_{i j 1}}+\left\|P_{i 3}\right\| L_{H_{i j 2}}\right), & i \neq j
\end{array}\right.
$$

where $H_{i j 1}$ and $H_{i j 2}$ are, respectively, the first $p_{i}$ and the last $n_{i}-p_{i}$ components of $H_{i j}\left(x_{j}\right)$, and $P_{i 2}, P_{i 3}$ and $Q_{i 3}$ are defined by (10).

Proof: From Assumption $3, C_{i}=\left[\begin{array}{ll}I_{p_{i}} & 0\end{array}\right]$ and the partition (10) of $P_{i}$, it follows that

$$
\begin{aligned}
P_{i 2}^{\tau} D_{i 1}+P_{i 3} D_{i 2} & =0, \\
P_{i 2}^{\tau} E_{i j 1}+P_{i 3} E_{i j 2} & =0 \quad(i \neq j)
\end{aligned}
$$

Introduce a nonsingular coordinate transformation $z_{i}=\hat{T}_{i} x_{i}$ defined by

$$
\hat{T}_{i}:\left\{\begin{array}{l}
z_{i 1}=x_{i 1} \\
z_{i 2}=P_{i 3}^{-1} P_{i 2}^{\tau} x_{i 1}+x_{i 2}
\end{array}\right.
$$

Since (12)-(13) implies $P_{i 3}^{-1} P_{i 2}^{\tau} D_{i 1}+D_{i 2}=0$ and $P_{i 3}^{-1} P_{i 2}^{\tau} E_{i j 1}+E_{i j 2}=0$, it follows from (8)-(9) that in the new coordinates $z=\operatorname{col}\left(z_{i 1}, \ldots, z_{i N}\right)$, system (1)-(2) is described by

$$
\begin{gathered}
\dot{z}_{i 1}=\left(A_{i 1}-A_{i 2} P_{i 3}^{-1} P_{i 2}^{\tau}\right) z_{i 1}+A_{i 2} z_{i 2}+B_{i 1} u_{i}+ \\
D_{i 1} \Delta \tilde{f}_{i}+\sum_{\substack{j=1 \\
j \neq i}}^{N}\left(H_{i j 1}+E_{i j 1} \Delta \widetilde{H}_{i j}\right) \\
\dot{z}_{i 2}=\left(P_{i 3}^{-1} P_{i 2}^{\tau}\left(A_{i 1}-A_{i 2} P_{i 3}^{-1} P_{i 2}^{\tau}\right)+A_{i 3}-\right. \\
\left.A_{i 4} P_{i 3}^{-1} P_{i 2}^{\tau}\right) z_{i 1}+\left(A_{i 4}+P_{i 3}^{-1} P_{i 2}^{\tau} A_{i 2}\right) z_{i 2} \\
+\left(P_{i 3}^{-1} P_{i 2}^{\tau} B_{i 1}+B_{i 2}\right) u_{i}+ \\
\sum_{\substack{j=1 \\
j \neq i}}^{N}\left[P_{i 3}^{-1} P_{i 2}^{\tau} H_{i j 1}\left(y_{j}, \nu_{j}\right)+H_{i j 2}\left(y_{j}, \nu_{j}\right)\right] \\
y_{i}=z_{i 1}
\end{gathered}
$$

where $\nu_{j}=z_{j 2}-P_{j 3}^{-1} P_{j 2}^{\tau} z_{j 1}$. From the above, (14), and $\hat{x}_{i 2}=-P_{i 3}^{-1} P_{i 2}^{\tau} y_{i}+\hat{z}_{i 2}$, it follows that

$$
x_{i 2}-\hat{x}_{i 2}=x_{i 2}+P_{i 3}^{-1} P_{i 2}^{\tau} y_{i}-\hat{z}_{i 2}=z_{i 2}-\hat{z}_{i 2}
$$

It is only required to prove that $\left\|z_{i 2}-\hat{z}_{i 2}\right\| \leq$ $\alpha_{1} \exp \left\{-\alpha_{2} t\right\}$ for positive constants $\alpha_{1}$ and $\alpha_{2}$.

Let $e_{i}=z_{i 2}-\hat{z}_{i 2}$. Substituting from the dynamics,

$$
\begin{aligned}
\dot{e}_{i}= & \left(A_{i 4}+P_{i 3}^{-1} P_{i 2}^{\tau} A_{i 2}\right) e_{i}+ \\
& \sum_{\substack{j=1 \\
j \neq i}}^{N}\left\{P_{i 3}^{-1} P_{i 2}^{\tau}\left[H_{i j 1}\left(y_{j}, \nu_{j}\right)-H_{i j 1}\left(y_{j}, \hat{\nu}_{j}\right)\right]\right. \\
+ & \left.H_{i j 2}\left(y_{j}, \nu_{j}\right)-H_{i j 2}\left(y_{j}, \hat{\nu}_{j}\right)\right\}
\end{aligned}
$$

where $\nu_{j}=z_{j 2}-P_{j 3}^{-1} P_{j 2}^{\tau} y_{j}$ and $\hat{\nu}_{j}=\hat{z}_{j 2}-$ $P_{j 3}^{-1} P_{j 2}^{\tau} y_{j}$. For system (15), consider a Lyapunov function candidate $V_{1}=\sum_{i=1}^{N} e_{i}^{\tau} P_{i 3} e_{i}$. Then, the time derivative of $V_{1}$ along the trajectories of system (15) is described by

$$
\begin{gathered}
\dot{V}_{1}=\sum_{i=1}^{N} e_{i}^{\tau}\left(\left[A_{i 4}+P_{i 3}^{-1} P_{i 2}^{\tau} A_{i 2}\right]^{\tau} P_{i 3}\right. \\
\left.+P_{i 3}\left[A_{i 4}+P_{i 3}^{-1} P_{i 2}^{\tau} A_{i 2}\right]\right) e_{i} \\
+2 \sum_{i=1}^{N} \sum_{\substack{j=1 \\
j \neq i}}^{N} e_{i}^{\tau}\left(P_{i 2}^{\tau}\left[H_{i j 1}\left(y_{j}, \nu_{j}\right)-H_{i j 1}\left(y_{j}, \hat{\nu}_{j}\right)\right]+\right. \\
\left.P_{i 3}\left[H_{i j 2}\left(y_{j}, \nu_{j}\right)-H_{i j 2}\left(y_{j}, \hat{\nu}_{j}\right)\right]\right)
\end{gathered}
$$

From (4), (10) and $C_{i}=\left[I_{p_{i}} \tau_{\tau} 0\right]$, it follows that

$$
\begin{gathered}
\left(P_{i 3}^{-1} P_{i 2}^{\tau} A_{i 2}+A_{i 4}\right)^{\tau} P_{i 3}+ \\
P_{i 3}\left(P_{i 3}^{-1} P_{i 2}^{\tau} A_{i 2}+A_{i 4}\right)=-Q_{i 3}
\end{gathered}
$$

Since Assumption 2 implies that both $H_{i j 1}$ and $H_{i j 2}$ are Lipschitz in their domain of definition, $L_{H_{i j 1}}$ and $L_{H_{i j 2}}$ are well defined. Then, substituting (17) into (16), it is observed from $\nu_{i}-\hat{\nu}_{i}=e_{i}$ that

$$
\begin{aligned}
\dot{V}_{1} \leq & -\sum_{i=1}^{N} e_{i}^{\tau} Q_{i 3} e_{i}+2 \sum_{i=1}^{N} \sum_{\substack{j=1 \\
j \neq i}}^{N}\left(\left\|P_{i 2}\right\| L_{H_{i j 1}}+\right. \\
& \left.\left\|P_{i 3}\right\| L_{H_{i j 2}}\right)\left\|e_{i}\right\|\left\|e_{j}\right\| \\
\leq & -\frac{1}{2}\left[\left\|e_{1}\right\| \cdots\left\|e_{N}\right\|\right]\left(W+W^{\tau}\right)\left[\left\|e_{1}\right\| \cdots\left\|e_{N}\right\|\right]^{\tau} \\
\leq & -\frac{\underline{\lambda}\left(W+W^{\tau}\right)}{2 \max _{i}\left\{\bar{\lambda}\left(P_{i 3}\right)\right\}} V_{1}
\end{aligned}
$$

This implies $V_{1} \leq\left(\left.V_{1}\right|_{t=0}\right) \exp \left\{-\frac{\underline{\lambda}\left(W+W^{\tau}\right)}{2 \max _{i}\left\{\bar{\lambda}\left(P_{i 3}\right)\right.}\right\}$. Then, from

$$
\min _{i}\left\{\underline{\lambda}\left(P_{i 3}\right)\right\}\left\|e_{i}\right\|^{2} \leq e_{i}^{\tau} P_{i 3} e_{i} \leq \sum_{i=1}^{N} e_{i}^{\tau} P_{i 3} e_{i}=V_{1}
$$

the conclusion follows if $\alpha_{1}>\sqrt{\frac{\left.V_{1}\right|_{t=0}}{\max _{i}\left\{\underline{\lambda}\left(P_{i 3}\right)\right\}}}$ and $\alpha_{2} \geq \frac{\underline{\lambda}\left(W+W^{\tau}\right)}{2 \max _{i}\left\{\bar{\lambda}\left(P_{i 3}\right)\right\}}$.

\section{SLIDING MODE ANALYSIS}

For system (1)-(2), consider the sliding surface

$$
\begin{aligned}
\sigma & \equiv: \operatorname{col}\left(\sigma_{1}, \sigma_{2}, \ldots, \sigma_{N}\right)=0 \\
\sigma_{i}\left(y_{i}, \hat{x}_{i 2}\right) & =S_{i 1} y_{i}+S_{i 2} \hat{x}_{i 2}
\end{aligned}
$$

where $\hat{x}_{i 2}$ is the compensator state in Theorem 1 , and $S_{i 1} \in \mathbb{R}^{m_{i} \times p_{i}}$ and $S_{i 2} \in \mathbb{R}^{m_{i} \times\left(n_{i}-p_{i}\right)}$ are the design parameters. 
As in the proof of Theorem 1, let $e_{i}=x_{i 2}-\hat{x}_{i 2}$ and define $S_{i}=\left[\begin{array}{ll}S_{i 1} & S_{i 2}\end{array}\right]$. In the new coordinate system $\left(x_{i}, e_{i}\right)$, the sliding function matrices

$$
\sigma_{i}=\left[\begin{array}{ll}
S_{i 1} & S_{i 2}
\end{array}\right] x_{i}-S_{i 2} e_{i}=S_{i} x_{i}-S_{i 2} e_{i} .
$$

The matrices $S_{i}$ can be chosen using any existing state feedback sliding mode design approach on the pairs $\left(A_{i}, B_{i}\right)$ such that:

i) the matrices $S_{i} B_{i}$ are nonsingular;

ii) the matrices $A_{e q i} \equiv: A_{i}-B_{i}\left(S_{i} B_{i}\right)^{-1} S_{i} A_{i}$ have $n_{i}-m_{i}$ eigenvalues which lie in the open left-half plane.

During a sliding motion, both $\sigma_{i}=0$ and $\dot{\sigma}_{i}=0$. From (1), (15), (20) and $\dot{\sigma}_{i}=0$, the equivalent control (Utkin 1978) necessary to maintain a sliding motion is given by

$$
\begin{gathered}
u_{i e q}=-\left(S_{i} B_{i}\right)^{-1}\left\{S_{i} A_{i} x_{i}-S_{i 2}\left(A_{i 4}+P_{i 3}^{-1} P_{i 2}^{\tau} A_{i 2}\right) e_{i}\right. \\
+S_{i} \Delta f_{i}+\sum_{\substack{j=1 \\
j \neq i}}^{N} S_{i}\left(H_{i j}\left(x_{j}\right)+\Delta H_{i j}\right)- \\
S_{i 2} \sum_{\substack{j=1 \\
j \neq i}}^{N}\left(P_{i 3}^{-1} P_{i 2}^{\tau}\left(H_{i j 1}\left(y_{j}, x_{j 2}\right)-H_{i j 1}\left(y_{j}, \hat{x}_{j 2}\right)\right)\right. \\
\left.\left.\quad+H_{i j 2}\left(y_{j}, x_{j 2}\right)-H_{i j 2}\left(y_{j}, \hat{x}_{j 2}\right)\right)\right\}
\end{gathered}
$$

When system (1)-(2) is restricted to the sliding surface (18), it follows by applying the control above to system (1) that the associated dynamics are given by

$$
\begin{gathered}
{\left[\begin{array}{c}
\dot{x}_{i} \\
\dot{e}_{i}
\end{array}\right]=\left[\begin{array}{cc}
A_{\text {eqi }} & B_{i}\left(S_{i} B_{i}\right)^{-1} S_{i 2}\left(A_{i 4}+P_{i 3}^{-1} P_{i 2}^{\tau} A_{i 2}\right) \\
0 & A_{i 4}+P_{i 3}^{-1} P_{i 2}^{\tau} A_{i 2}
\end{array}\right]\left[\begin{array}{c}
x_{i} \\
e_{i}
\end{array}\right]} \\
+\left[\begin{array}{c}
\left(I_{n_{i}}-B_{i}\left(S_{i} B_{i}\right)^{-1} S_{i}\right)\left(\Delta f_{i}+\sum_{\substack{j=1 \\
j \neq i}}^{N}\left(H_{i j}+\Delta H_{i j}\right)\right) \\
0
\end{array}\right] \\
+\left[\begin{array}{c}
B_{i}\left(S_{i} B_{i}\right)^{-1} S_{i 2} \\
I_{n_{i}-p_{i}}
\end{array}\right] \times \sum_{\substack{j=1 \\
j \neq i}}^{N}\left\{P _ { i 3 } ^ { - 1 } P _ { i 2 } ^ { \tau } \left(H_{i j 1}\left(y_{j}, x_{j 2}\right)-\right.\right. \\
\left.\left.H_{i j 1}\left(y_{j}, \hat{x}_{j 2}\right)\right)+H_{i j 2}\left(y_{j}, x_{j 2}\right)-H_{i j 2}\left(y_{j}, \hat{x}_{j 2}\right)\right\}
\end{gathered}
$$

where $A_{e q i}=A_{i}-B_{i}\left(S_{i} B_{i}\right)^{-1} S_{i} A_{i}$. Since $S_{i} B_{i}$ is nonsingular, matrix $S_{i}$ is full row rank and thus there exist nonsingular matrices $T_{i 1} \in \mathbb{R}^{n_{i} \times n_{i}}$ and $T_{i 2} \in \mathbb{R}^{m_{i} \times m_{i}}$ such that

$$
T_{i 2} S_{i} T_{i 1}=\left[\begin{array}{ll}
I_{m_{i}} & 0
\end{array}\right]
$$

In order to further analyse the stability of the sliding mode, it is required to derive a reduced order representation. The coordinate transformation $\operatorname{col}\left(\xi_{i}, \eta_{i}\right)=T_{i 1}^{-1} x_{i}$ is introduced, where $\xi_{i} \in$ $\mathbb{R}^{m_{i}}$ and $T_{i 1}$ is determined by (21). Then, noticing the condition ii), it follows that in the new coordinates $\left(\xi_{i}, \eta_{i}, e_{i}\right)$, the system is described by the equations

$$
\left[\begin{array}{c}
\dot{\xi}_{i} \\
\dot{\eta}_{i} \\
\dot{e}_{i}
\end{array}\right]=\left[\begin{array}{ccc}
0 & 0 & * \\
\widetilde{A}_{i 1} & \widetilde{A}_{i 2} & \widetilde{A}_{i 3} \\
0 & 0 & A_{i 4}+P_{i 3}^{-1} P_{i 2}^{\tau} A_{i 2}
\end{array}\right]\left[\begin{array}{l}
\xi_{i} \\
\eta_{i} \\
e_{i}
\end{array}\right]
$$

$$
+\left[\begin{array}{c}
* \\
\Delta f_{i 1} \\
0
\end{array}\right]+\sum_{\substack{j=1 \\
j \neq i}}^{N}\left[\begin{array}{c}
* \\
\Pi_{i j} \\
0
\end{array}\right]+\sum_{\substack{j=1 \\
j \neq i}}^{N}\left[\begin{array}{c}
* \\
\Theta_{i j 1} \\
\Theta_{i j 2}
\end{array}\right]
$$

where $\widetilde{A}_{i 2} \in \mathbb{R}^{\left(n_{i}-m_{i}\right) \times\left(n_{i}-m_{i}\right)}, \widetilde{A}_{i 3} \in \mathbb{R}^{\left(n_{i}-m_{i}\right) \times\left(n_{i}-p_{i}\right)}$, and

$$
\left[\begin{array}{cc}
0 & 0 \\
\widetilde{A}_{i 1} & \widetilde{A}_{i 2}
\end{array}\right]=T_{i 1}^{-1} A_{e q i} T_{i 1}
$$

The notation $*$ denotes items which do not play a role in the subsequent analysis; $\Delta f_{i 1}, \Pi_{i j}$ and $\Theta_{i j 1}$ are the last $n_{i}-m_{i}$ components of

$$
\begin{array}{r}
T_{i 1}^{-1}\left(I_{n_{i}}-B_{i}\left(S_{i} B_{i}\right)^{-1} S_{i}\right) \Delta f_{i}, \\
T_{i 1}^{-1}\left(I_{n_{i}}-B_{i}\left(S_{i} B_{i}\right)^{-1} S_{i}\right)\left(H_{i j}+\Delta H_{i j}\right)
\end{array}
$$

and

$$
\begin{gathered}
T_{i 1}^{-1} B_{i}\left(S_{i} B_{i}\right)^{-1} S_{i 2}\left\{P _ { i 3 } ^ { - 1 } P _ { i 2 } ^ { \tau } \left(H_{i j 1}\left(y_{j}, x_{j 2}\right)-\right.\right. \\
\left.\left.H_{i j 1}\left(y_{j}, \hat{x}_{j 2}\right)\right)+H_{i j 2}\left(y_{j}, x_{j 2}\right)-H_{i j 2}\left(y_{j}, \hat{x}_{j 2}\right)\right\}
\end{gathered}
$$

respectively, and

$$
\begin{aligned}
\Theta_{i j 2}= & P_{i 3}^{-1} P_{i 2}^{\tau}\left(H_{i j 1}\left(y_{j}, x_{j 2}\right)-H_{i j 1}\left(y_{j}, \hat{x}_{j 2}\right)\right) \\
& +H_{i j 2}\left(y_{j}, x_{j 2}\right)-H_{i j 2}\left(y_{j}, \hat{x}_{j 2}\right)
\end{aligned}
$$

From (21), it follows that

$$
\begin{aligned}
\sigma_{i} & =S_{i} x_{i}-S_{i 2} e_{i}=T_{i 2}^{-1}\left[\begin{array}{ll}
I_{m_{i}} & 0
\end{array}\right]\left[\begin{array}{c}
\xi_{i} \\
\eta_{i}
\end{array}\right]-S_{i 2} e_{i} \\
& =T_{i 2}^{-1} \xi_{i}-S_{i 2} e_{i}
\end{aligned}
$$

This implies that in the new coordinate system $\left(\xi_{i}, \eta_{i}, e_{i}\right), \sigma_{i}=0$ can be depicted by $\xi_{i}=T_{i 2} S_{i 2} e_{i}$. Consequently, when the system is restricted to the sliding surface (18), it can be described in coordinate system $\left(\xi_{i}, \eta_{i}, e_{i}\right)$ by

$$
\begin{aligned}
{\left[\begin{array}{c}
\dot{\eta}_{i} \\
\dot{e}_{i}
\end{array}\right]=} & {\left[\begin{array}{cc}
\widetilde{A}_{i 2} & \widetilde{A}_{i 3}+\widetilde{A}_{i 1} T_{i 2} S_{i 2} \\
0 & A_{i 4}+P_{i 3}^{-1} P_{i 2}^{\tau} A_{i 2}
\end{array}\right]\left[\begin{array}{c}
\eta_{i} \\
e_{i}
\end{array}\right]+} \\
& {\left[\begin{array}{c}
\Delta f_{i 1} \\
0
\end{array}\right]+\sum_{\substack{j=1 \\
j \neq i}}^{N}\left[\begin{array}{c}
\Pi_{i j} \\
0
\end{array}\right]+\sum_{\substack{j=1 \\
j \neq i}}^{N}\left[\begin{array}{l}
\Theta_{i j 1} \\
\Theta_{i j 2}
\end{array}\right] }
\end{aligned}
$$

From condition ii) and (23), the matrix $\widetilde{A}_{i 2}$ has $n_{i}-m_{i}$ negative eigenvalues. This implies that for any $\widetilde{Q}_{i}>0$, the Lyapunov equations

$$
\widetilde{A}_{i 2}^{\tau} \widetilde{P}_{i}+\widetilde{P}_{i} \widetilde{A}_{i 2}=-\widetilde{Q}_{i}
$$

have unique solutions $\widetilde{P}_{i}>0$.

From (3) and Assumption 2, there exist continuous functions $\varphi_{i 1}, \varphi_{i 2}, \psi_{i j}$ and $\chi_{i j}$ such that

$$
\begin{gathered}
\left\|\widetilde{P}_{i} \Delta f_{i 1}\right\| \leq \varphi_{i 1}\left(\eta_{i}, e_{i}\right)\left\|\eta_{i}\right\|+\varphi_{i 2}\left(\eta_{i}, e_{i}\right)\left\|e_{i}\right\| \\
\left\|\widetilde{P}_{i}\left(\Pi_{i j}+\Theta_{i j 1}\right)\right\| \leq \psi_{i j}\left(\eta_{j}, e_{j}\right)\left\|\eta_{j}\right\| \\
+\chi_{i j}\left(\eta_{j}, e_{j}\right)\left\|e_{j}\right\|
\end{gathered}
$$

where $\widetilde{P}_{i}$ satisfies $(26)$.

Theorem 2. Under Assumptions 1-3, the sliding mode dynamics are asymptotically stable if there exists a domain of the origin $\mathcal{O}_{i} \subset \mathbb{R}^{2 n_{i}-m_{i}-p_{i}}$ such that for $\operatorname{col}\left(\eta_{1}, e_{1}, \ldots, \eta_{N}, e_{N}\right) \in \mathcal{O}_{1} \times \cdots \times$ $\mathcal{O}_{N}$, the matrix $M^{\tau}+M$ is positive definite with $M \in \mathbb{R}^{2 N \times 2 N}$ defined by 


$$
\left[\begin{array}{cccc|cccc}
\underline{\lambda}\left(\widetilde{Q}_{1}\right)-2 \varphi_{11} & -2 \psi_{12} & \cdots & -2 \psi_{1 N} & -2\left(\varphi_{12}+\varpi_{1}\right) & -2 \chi_{12} & \cdots & -2 \chi_{1 N} \\
-2 \psi_{21} & \underline{\lambda}\left(\widetilde{Q}_{2}\right)-2 \varphi_{21} & \ddots & \vdots & -2 \chi_{21} & -2\left(\varphi_{22}+\varpi_{2}\right) & \ddots & \vdots \\
\vdots & \ddots & \ddots & -2 \psi_{(N-1) N} & \vdots & \ddots & \ddots & -2 \chi_{(N-1) N} \\
-2 \psi_{N 1} & \ldots & -2 \psi_{N(N-1)} & \underline{\lambda}\left(\widetilde{Q}_{N}\right)-2 \varphi_{N 1} & -2 \chi_{N 1} & \ldots \ldots & -2 \chi_{N(N-1)}-2\left(\phi_{N 2}+\varpi_{N}\right) \\
\hline-2\left(\varphi_{12}+\varpi_{1}\right) & -2 \chi_{12} & \cdots & -2 \chi_{1 N} & \underline{\lambda}\left(Q_{13}\right) & -2 \kappa_{12} & \ddots & \ddots \\
-2 \chi_{21} & -2\left(\varphi_{22}+\varpi_{2}\right) & \ddots & \vdots & -2 \kappa_{21} & \underline{\lambda}\left(Q_{23}\right) & \ddots \\
\vdots & \ddots & \ddots & -2 \chi_{(N-1) N} & \ddots & \ddots & -2 \kappa_{(N-1) N} \\
-2 \chi_{N 1} & \cdots & -2 \chi_{N(N-1)}-2\left(\varphi_{N 2}+\varpi_{N}\right) & -2 \kappa_{N 1} & \cdots & -2 \kappa_{N(N-1)} & \underline{\lambda}\left(Q_{N 3}\right)
\end{array}\right]
$$

where $\varphi_{i 1}, \varphi_{i 2}, \psi_{i j}$ and $\chi_{i j}$ are determined by the equations above, $\kappa_{i j}: \equiv\left(\left\|P_{i 2}\right\| L_{H_{i j 1}}+\left\|P_{i 3}\right\| L_{H_{i j 2}}\right)$ and $\varpi_{i}: \equiv\left\|\widetilde{P}_{i}\left(\widetilde{A}_{i 3}+\widetilde{A}_{i 1} T_{i 2} S_{i 2}\right)\right\|$.

Proof: Consider a Lyapunov function $V=$ $\sum_{i=1}^{N}\left(\eta_{i}^{\tau} \widetilde{P}_{i} \eta_{i}+e_{i}^{\tau} P_{i 3} e_{i}\right)$. Then, the time derivative of $V$ along the trajectories of the system is given by

$$
\begin{aligned}
\dot{V}= & -\sum_{i=1}^{N} \eta_{i}^{\tau} \widetilde{Q}_{i} \eta_{i}+2 \sum_{i=1}^{N} \eta_{i}^{\tau} \widetilde{P}_{i}\left(\widetilde{A}_{i 3}+\widetilde{A}_{i 1} T_{i 2} S_{i 2}\right) e_{i} \\
& +2 \sum_{i=1}^{N} \sum_{\substack{j=1 \\
j \neq i}}^{N} \eta_{i}^{\tau} \widetilde{P}_{i}\left(\Pi_{i j}+\Theta_{i j 1}\right)+2 \sum_{i=1}^{N} \eta_{i}^{\tau} \widetilde{P}_{i} \Delta f_{i 1} \\
& -\sum_{i=1}^{N} e_{i}^{\tau} Q_{i 3} e_{i}+2 \sum_{i=1}^{N} \sum_{\substack{j=1 \\
j \neq i}}^{N} e_{i}^{\tau} P_{i 3} \Theta_{i j 2} \\
\leq & -\sum_{i=1}^{N}\left(\underline{\lambda}_{(}\left(\widetilde{Q}_{i}\right)\left\|\eta_{i}\right\|^{2}+\underline{\lambda}\left(Q_{i 3}\right)\left\|e_{i}\right\|^{2}\right) \\
& +2 \sum_{i=1}^{N}\left\|\widetilde{P}_{i}\left(\widetilde{A}_{i 3}+\widetilde{A}_{i 1} T_{i 2} S_{i 2}\right)\right\|\left\|e_{i}\right\|\left\|\eta_{i}\right\| \\
& +2 \sum_{i=1}^{N}\left\|\widetilde{P}_{i} \Delta f_{i 1}\right\|\left\|\eta_{i}\right\|+2 \sum_{i=1}^{N} \sum_{\substack{j=1 \\
j \neq i}}^{N}\{ \\
& \left.\left\|\widetilde{P}_{i}\left(\Pi_{i j}+\Theta_{i j 1}\right)\right\|\left\|\eta_{i}\right\|+\left\|P_{i 3} \Theta_{i j 2}\right\|\left\|e_{i}\right\|\right\}(27)
\end{aligned}
$$

where (17) and (26) are used above. From (24),

$$
\begin{gathered}
\left\|P_{i 3} \Theta_{i j 2}\right\|=\| P_{i 2}^{\tau}\left(H_{i j 1}\left(y_{j}, x_{j 2}\right)-H_{i j 1}\left(y_{j}, \hat{x}_{j 2}\right)\right)+ \\
P_{i 3}\left(H_{i j 2}\left(y_{j}, x_{j 2}\right)-H_{i j 2}\left(y_{j}, \hat{x}_{j 2}\right)\right) \| \\
\leq\left(\left\|P_{i 2}\right\|_{H_{i j 1}}+\left\|P_{i 3}\right\|_{H_{i j 2}}\right)\left\|e_{j}\right\|=\kappa_{i j}\left\|e_{j}\right\|
\end{gathered}
$$

Then, from the above, and the definitions of the functions $\varphi_{i 1}, \varphi_{i 2}, \psi_{i j}$ and $\chi_{i j}$ :

$$
\begin{gathered}
\dot{V} \leq-\sum_{i=1}^{N}\left(\underline{\lambda}\left(\widetilde{Q}_{i}\right)-2 \varphi_{i 1}\right)\left\|\eta_{i}\right\|^{2}-\sum_{i=1}^{N} \underline{\lambda}\left(Q_{i 3}\right)\left\|e_{i}\right\|^{2} \\
+2 \sum_{i=1}^{N}\left(\varphi_{i 2}+\varpi_{i}\right)\left\|\eta_{i}\right\|\left\|e_{i}\right\| \\
+2 \sum_{\substack { i=1 \\
\begin{subarray}{c}{j=1 \\
j \neq i{ i = 1 \\
\begin{subarray} { c } { j = 1 \\
j \neq i } }\end{subarray}}^{N}\left\{\psi_{i j}\left\|\eta_{i}\right\|\left\|\eta_{j}\right\|+\chi_{i j}\left\|\eta_{i}\right\|\left\|e_{j}\right\|\right. \\
\left.+\kappa_{i j}\left\|e_{i}\right\|\left\|e_{j}\right\|\right\} \\
=\frac{1}{2}\left[\left\|\eta_{1}\right\| \cdots\left\|\eta_{N}\right\|\left\|e_{1}\right\| \cdots\left\|e_{N}\right\|\right] \\
\left(M^{\tau}+M\right)\left[\left\|\eta_{1}\right\| \cdots\left\|\eta_{N}\right\|\left\|e_{1}\right\| \cdots\left\|e_{N}\right\|\right]^{\tau}
\end{gathered}
$$

Hence, the conclusion follows by the positive definiteness of $M^{\tau}+M$.

\section{SLIDING MODE CONTROLLER}

For the system (1)-(2) with the designed composite sliding surface (18), construct the following sliding mode control

$$
\begin{aligned}
u_{i}= & -\left(S_{i} B_{i}\right)^{-1}\left\{\left(S_{i 1} A_{i 1}+S_{i 2} A_{i 3}\right) y_{i}+\left(S_{i 1} A_{i 2}+\right.\right. \\
& \left.S_{i 2} A_{i 4}\right) \hat{x}_{i 2}+\left(\left\|S_{i} D_{i}\right\| \rho_{i}\left(y_{i}, t\right) \gamma_{i}\left(y_{i}, \hat{x}_{i 2}, t\right)\right. \\
+ & K_{i}\left(y_{i}, t\right)+\sum_{\substack{j=1 \\
j \neq i}}^{N}\left(\left\|S_{j} E_{j i}\right\| \vartheta_{j i}\left(y_{i}, t\right) \zeta_{j i}\left(y_{i}, \hat{x}_{i 2}, t\right)+\right. \\
& \left.\left.\left.\left.\left\|S_{j} H_{j i}\left(y_{i}, \hat{x}_{i 2}\right)\right\|\right)\right)\right) \frac{\sigma_{i}}{\left\|\sigma_{i}\right\|}\right\}
\end{aligned}
$$

where $\sigma_{i}$ is defined by (19), and $K_{i}\left(y_{i}, t\right)$ is the control gain to be determined later. The control law is decentralised and only depends on the $\hat{x}_{i 2}$ and the system output $y_{i}$. It is necessary to show that the above control can drive the system (1)-(2) to the sliding surface (18) and maintain sliding. It is required to prove that the composite reachability condition (see (Hsu 1997))

$$
\sum_{i=1}^{N} \frac{\sigma_{i}^{\tau}\left(y_{i}, \hat{x}_{i 2}\right) \dot{\sigma}_{i}\left(y_{i}, \hat{x}_{i 2}\right)}{\left\|\sigma_{i}\left(y_{i}, \hat{x}_{i 2}\right)\right\|}<0 .
$$

is satisfied, where $\sigma_{i}\left(y_{i}, \hat{x}_{i 2}\right)$ defined by (19) is the sliding function for the $i$-th subsystem.

Theorem 3. Under Assumptions 1-3 with (11) satisfied, the controller drives the system (1)-(2) to the composite sliding surface (18) and maintain a sliding motion thereafter if the control gains $K_{i}$ are chosen such that

$$
\begin{gathered}
K_{i}\left(y_{i}, t\right)>\alpha_{1} \exp \left\{-\alpha_{2} t\right\}\left\{\left\|S_{i 1} A_{i 2}+S_{i 2} A_{i 4}\right\|+\right. \\
L_{\gamma_{i}}\left\|S_{i} D_{i}\right\| \rho_{i}\left(y_{i}, t\right)+\left\|S_{i 2} P_{i 3}^{-1}\left(P_{i 2}^{\tau} A_{i 2}+P_{i 3} A_{i 4}\right)\right\| \\
\quad+\sum_{\substack{j=1 \\
j \neq i}}^{N}\left(\left\|S_{j}\right\| L_{H_{j i}}+\left\|S_{j} E_{j i} \vartheta_{j i}\right\| L_{\zeta_{j i}}+\right. \\
\left.\left.\left\|S_{i 2} P_{i 3}^{-1}\right\|\left(\left\|P_{i 2}\right\| L_{H_{i j 1}}+\left\|P_{i 3}\right\| L_{H_{i j 2}}\right)\right)\right\}
\end{gathered}
$$

with the constants $\alpha_{1}$ and $\alpha_{2}$ determined by (11). Proof: From the proof of Theorem 1 the error dynamics in (15) can be rewritten $N$

$$
\begin{aligned}
\dot{e}_{i}= & P_{i 3}^{-1}\left(P_{i 2}^{\tau} A_{i 2}+P_{i 3} A_{i 4}\right) e_{i}+\sum_{\substack{j=1 \\
j \neq i}}^{N} P_{i 3}^{-1} \times \\
& \left\{P_{i 2}^{\tau}\left(H_{i j 1}\left(y_{j}, x_{j 2}\right)-H_{i j 1}\left(y_{j}, \hat{x}_{j 2}\right)\right)\right. \\
& \left.+P_{i 3}\left(H_{i j 2}\left(y_{j}, x_{j 2}\right)-H_{i j 2}\left(y_{j}, \hat{x}_{j 2}\right)\right)\right\}
\end{aligned}
$$

From (20), (1) and (29) 


$$
\begin{aligned}
\dot{\sigma}_{i}= & S_{i} A_{i} x_{i}+S_{i} B_{i} u_{i}+S_{i} \Delta f_{i}+\sum_{\substack{j=1 \\
j \neq i}}^{N} S_{i}\left(H_{i j}\left(x_{j}\right)+\right. \\
& \left.\Delta H_{i j}\left(x_{j}, t\right)\right)-S_{i 2} P_{i 3}^{-1}\left(P_{i 2}^{\tau} A_{i 2}+P_{i 3} A_{i 4}\right) e_{i} \\
& -\sum_{\substack{j=1 \\
j \neq i}}^{N} S_{i 2} P_{i 3}^{-1}\left\{P_{i 2}^{\tau}\left(H_{i j 1}\left(y_{j}, x_{j 2}\right)-H_{i j 1}\left(y_{j}, \hat{x}_{j 2}\right)\right)+\right. \\
& \left.P_{i 3}\left(H_{i j 2}\left(y_{j}, x_{j 2}\right)-H_{i j 2}\left(y_{j}, \hat{x}_{j 2}\right)\right)\right\}
\end{aligned}
$$

Then, substituting the proposed control $u_{i}$ into the above equation,

$$
\begin{gathered}
\sum_{i=1}^{N} \frac{\sigma_{i}^{\tau} \dot{\sigma}_{i}}{\left\|\sigma_{i}\right\|}=\sum_{i=1}^{N} \frac{\sigma_{i}^{\tau}}{\left\|\sigma_{i}\right\|}\left\{\left(S_{i} A_{i} x_{i}-\left(S_{i 1} A_{i 1}+S_{i 2} A_{i 3}\right) y_{i}-\right.\right. \\
\left.\left(S_{i 1} A_{i 2}+S_{i 2} A_{i 4}\right) \hat{x}_{i 2}\right) \\
\left.-S_{i 2} P_{i 3}^{-1}\left(P_{i 2}^{\tau} A_{i 2}+P_{i 3} A_{i 4}\right) e_{i}\right\}+\sum_{i=1}^{N}\left(\frac{\sigma_{i}^{\tau}}{\left\|\sigma_{i}\right\|} S_{i} \Delta f_{i}\right. \\
\left.-\left\|S_{i} D_{i}\right\| \rho_{i}\left(y_{i}, t\right) \gamma_{i}\left(y_{i}, \hat{x}_{i 2}, t\right)\right)-\sum_{i=1}^{N} K_{i} \\
+\sum_{\substack{i=1 \\
j}}^{N} \sum_{\substack{j=1 \\
j \neq i}}^{N} \frac{\sigma_{i}^{\tau}}{\left\|\sigma_{i}\right\|}\left(S_{i}\left[H_{i j}+\Delta H_{i j}\right]-\frac{\sigma_{i}}{\left\|\sigma_{i}\right\|} \times\right. \\
\left.-\sum_{\substack{i=1 \\
S_{j}}}^{N} \sum_{j i}^{N}\left(y_{i}, \hat{x}_{i 2}\right)\|+\| S_{j 2} E_{j i} S_{i 3}^{-1}\left\{\vartheta_{j i}\left(y_{i}, t\right) \zeta_{j i}\left(y_{i}, \hat{x}_{i 2}, t\right)\right]\right) \\
+P_{i 3}\left[H_{i j 1}\left(y_{j}, x_{j 2}\right)-H_{i j 1}\left(y_{j}, \hat{x}_{j 2}\right)\right]
\end{gathered}
$$

Using the previous partition of $A_{i}$ in (8) and $S_{i}=\left[\begin{array}{ll}S_{i 1} & S_{i 2}\end{array}\right]$, it follows that

$$
\begin{gathered}
S_{i} A_{i} x_{i}-\left(S_{i 1} A_{i 1}+S_{i 2} A_{i 3}\right) y_{i}-\left(S_{i 1} A_{i 2}+S_{i 2} A_{i 4}\right) \hat{x}_{i 2} \\
=\left[\begin{array}{ll}
S_{i 1} & S_{i 2}
\end{array}\right]\left[\begin{array}{cc}
A_{i 1} & A_{i 2} \\
A_{i 3} & A_{i 4}
\end{array}\right]\left[\begin{array}{l}
x_{i 1} \\
x_{i 2}
\end{array}\right]-\left(S_{i 1} A_{i 1}+S_{i 2} A_{i 3}\right) x_{i 1} \\
-\left(S_{i 1} A_{i 2}+S_{i 2} A_{i 4}\right) \hat{x}_{i 2} \\
=\left(S_{i 1} A_{i 2}+S_{i 2} A_{i 4}\right) e_{i}
\end{gathered}
$$

From Assumption 2

$$
\begin{aligned}
& \frac{\sigma_{i}^{\tau}}{\left\|\sigma_{i}\right\|} S_{i} \Delta f_{i}-\left\|S_{i} D_{i}\right\| \rho_{i}\left(y_{i}, t\right) \gamma_{i}\left(y_{i}, \hat{x}_{i 2}, t\right) \leq\left\|S_{i} D_{i}\right\| \times \\
& \left\|\Delta \widetilde{f}_{i}\right\|-\left\|S_{i} D_{i}\right\| \rho_{i}\left(y_{i}, t\right) \gamma_{i}\left(y_{i}, \hat{x}_{i 2}, t\right) \\
& \leq \rho_{i}\left(y_{i}, t\right)_{\gamma_{i}}\left\|S_{i} D_{i}\right\|\left\|e_{i}\right\| \\
& \text { and as } \sum_{i=1}^{N} \sum_{\substack{j=1 \\
j \neq i}}^{N} a_{i j}=\sum_{i=1}^{N} \sum_{\substack{j=1 \\
j \neq i}}^{N} a_{j i} \text { : } \\
& \sum_{i=1}^{N} \sum_{\substack{j=1 \\
j \neq i}}^{N} \frac{\sigma_{i}^{\tau}}{\left\|\sigma_{i}\right\|}\left(S_{i}\left[H_{i j}\left(x_{j}\right)+\Delta H_{i j}\left(x_{j}, t\right)\right]-\frac{\sigma_{i}}{\left\|\sigma_{i}\right\|} \times\right. \\
& \left.\left[\left\|S_{j} H_{j i}\left(y_{i}, \hat{x}_{i 2}\right)\right\|+\left\|S_{j} E_{j i}\right\| \vartheta_{j i} \zeta_{j i}\left(y_{i}, \hat{x}_{i 2}, t\right)\right]\right) \\
& =\sum_{i=1}^{N} \sum_{\substack{j=1 \\
j \neq i}}^{N}\left\{\frac{\sigma_{i}^{\tau}}{\left\|\sigma_{i}\right\|} S_{i} H_{i j}\left(x_{j}\right)-\left\|S_{j} H_{j i}\left(y_{i}, \hat{x}_{i 2}\right)\right\|+\right. \\
& \left.\frac{\sigma_{i}^{\tau}}{\left\|\sigma_{i}\right\|} S_{i} E_{i j} \Delta \widetilde{H}_{i j}-\left\|S_{j} E_{j i}\right\| \vartheta_{j i} \zeta_{j i}\left(y_{i}, \hat{x}_{i 2}, t\right)\right\} \\
& \leq \sum_{i=1}^{N} \sum_{\substack{j=1 \\
j \neq i}}^{N}\left\{L_{H_{i j}}\left\|S_{i}\right\|\left\|e_{j}\right\|+\left\|S_{j} E_{j i}\right\| \vartheta_{j i}\left(y_{i}, t\right) \times\right.
\end{aligned}
$$

$$
\begin{gathered}
\left.\zeta_{j i}\left(x_{i}, t\right)-\left\|S_{j} E_{j i}\right\| \vartheta_{j i} \zeta_{j i}\left(y_{i}, \hat{x}_{i 2}, t\right)\right\} \\
\left.\leq \sum_{i=1}^{N} \sum_{\substack{j=1 \\
j \neq i}}^{N}\left(\left\|S_{j}\right\| L_{H_{j i}}+\left\|S_{j} E_{j i}\right\| \vartheta_{j i}\left(y_{i}, t\right) L_{\zeta_{j i}}\right)\left\|e_{i}\right\| 31\right)
\end{gathered}
$$

Using the above, it follows from (11) that

$$
\begin{aligned}
& \sum_{i=1}^{N} \frac{\sigma_{i}^{\tau} \dot{\sigma}_{i}}{\left\|\sigma_{i}\right\|} \\
& \leq \sum_{i=1}^{N}\left\{\alpha _ { 1 } \operatorname { e x p } \{ - \alpha _ { 2 } t \} \left(\left\|S_{i 1} A_{i 2}+S_{i 2} A_{i 4}\right\|+\right.\right. \\
& \quad L_{\gamma_{i}} \rho_{i}\left\|S_{i} D_{i}\right\|+\left\|S_{i 2} P_{i 3}^{-1}\left(P_{i 2}^{\tau} A_{i 2}+P_{i 3} A_{i 4}\right)\right\| \\
& \quad+\sum_{\substack{j=1 \\
j \neq i}}^{N}\left(\left\|S_{j}\right\| L_{H_{j i}}+\left\|S_{j} E_{j i}\right\| \vartheta_{j i} L_{\zeta_{j i}}+\right. \\
& \left.\left.\quad\left\|S_{i 2} P_{i 3}^{-1}\right\|\left(\left\|P_{i 2}\right\| L_{H_{i j 1}}+\left\|P_{i 3}\right\| L_{H_{i j 2}}\right)\right)\right) \\
& \left.\left.\quad-K_{i}\left(y_{i}, t\right)\right)\right\}
\end{aligned}
$$

Hence, from the conclusion follows.

\section{CONCLUSION}

A dynamical decentralised output feedback control has been presented using sliding mode techniques. Equivalent control theory and a local coordinate transformation are exploited to establish the stability of the reduced-order sliding mode. Known interconnections are used in the control design which insures the composite reachability condition can be satisfied by the control law. The approach allows both nominal isolated subsystems and the overall nominal interconnected system to be nonminimum phase. The uncertainties are mismatched and have nonlinear bounds.

\section{REFERENCES}

Hsu, K. C. (1997). Decentralized variablestructure control design for uncertain largescale systems with series nonlinearities. Int. J. Control 68(6), 1231-1240.

Utkin, V. I. (1978). Sliding modes and their application to variable structure systems. Moscow: MIR Publication House.

Yan, X. G. and L. Xie (2003). Reduced-order control for a class of nonlinear similar interconnected systems with mismatched uncertainty. Automatica 39(1), 91-99.

Yan, X. G., C. Edwards and S. K. Spurgeon (2004). Decentralised robust sliding mode control for a class of nonlinear interconnected systems by static output feedback. Automatica 40(4), 613-620. 